\title{
Stage IIB Cervical Cancer AJCC v6 and v7
}

National Cancer Institute

\section{Source}

National Cancer Institute. Stage IIB Cervical Cancer AJCC v6 and v7. NCI Thesaurus. Code C6320.

Stage IIB includes: T2b, N0, M0. T2b: T umor with parametrial invasion. N0: No regional lymph node metastasis. M0: No distant metastasis. (AJCC 6th and 7th eds.) 